\title{
EBS/C proton spectra from a virgin diamond crystal
}

\author{
M. Erich a,*, M. Kokkoris b, S. Fazinić ${ }^{c}$, and S. Petrović a
}

\begin{abstract}
${ }^{a}$ Laboratory of Physics, Vinča Institute of Nuclear Sciences, University of Belgrade, PO Box 552, Belgrade, Serbia
${ }^{b}$ Department of Physics, National Technical University of Athens, Zografou Campus 157 80, Athens, Greece

c Laboratory for Ion Beam Interactions, Department of Experimental Physics, Institute Ruđer Bošković, Bijenička cesta 54, 10000 Zagreb, Croatia
\end{abstract}

\begin{abstract}
In the present work, elastic backscattering channeling spectra, EBS/C, of protons in a $<100>$ diamond crystal were experimentally and theoretically studied via a new computer simulation code. Proton incident energies for EBS/C spectra were in the energy range from $1.0 \mathrm{MeV}$ to $1.9 \mathrm{MeV}$. The energy range was chosen in order to explore a distinct strong resonance of the ${ }^{12} \mathrm{C}\left(\mathrm{p}, \mathrm{p}_{0}\right)^{12} \mathrm{C}$ elastic scattering at $1737 \mathrm{keV}$. The computer simulation code applied for the fitting of the experimental spectra in the random mode was compared with the corresponding SIMNRA results. In the channeling mode, it assumes a Gompertz type sigmoidal dechanneling function, which has two fitting parameters, $x_{c}$ and $k$, the dechanneling range and rate, respectively. It also uses $\alpha$, ratio of the channeling to random energy losses, as a fitting parameter. It was observed that $x_{c}$ increases, $k$ decreases and $\alpha$ stays relatively constant with the proton incident energy. These observations confirm the physical interpretation of the fitting parameters. Also, they constitute the basics for the further development of the code for the quantification of induced amorphization and depth profiling of implanted ions.
\end{abstract}

Key words: EBS/C, dechanneling, diamond, carbon, computer simulation

*Corresponding author: marko.erich@gmail.com (Marko Erich)

\section{Introduction}

Elastic backscattering channeling spectra, EBS/C, with the modification of the wellknown Rutherford backscattering, RBS/C, differential cross section term, offer a distinct opportunity of analyzing light impurities present in low concentrations in heavy crystalline matrices, which are otherwise obscured by the background yield. Usually, in such cases, the 
considerably slower and more complicated NRA technique is implemented, due to the lack of a comprehensive, reliable code for their quantitative analysis. The basic physical problems impeding the creation of such a code are closely related to the well-documented uncertainties of the stopping power in the channeling mode and to the accurate description of the dechanneling function.

The process of the dechanneling of ions, i.e. the change between the confined crystal propagation of ions into the random one, has been extensively studied since the discovery of channeling phenomena in crystals [1]. The full understanding of this process is important for a successful reproduction of the recorded backscattering channeling spectra, which are almost exclusively created by previously dechanneled ions [2-4]. The dechanneling process in crystals could be treated either analytically [5-8], or via computer based simulations [9-12]. Often, in computer simulations of EBS/C spectra (especially when following the binary collision approximation approach) an exponential dechanneling function is assumed [1], or deduced [13]. However, in some cases, quite significant discrepancies between experimental and computer simulated EBS/C spectra based on the exponential dechanneling function were observed [1]. Recently, experimental RBS/C spectra of helium in silicon were simulated using an exponential dechanneling function with the addition of one more parameter, the depth from which the dechanneling process starts [14], clearly showing the need for one more parameter for a successful experimental dechanneling spectra simulation and the inadequacy of a one-parameter exponential dechanneling function. In the case of $\mathrm{MeV}$ protons impinging upon $\mathrm{Si}<110>$, the twoparameter Gompertz type sigmoidal dechanneling function was introduced, showing a better agreement with experimental results in comparison with the corresponding exponential dechanneling function $[15,16]$.

Diamond is the allotropic modification of carbon, and it is best known for its extreme hardness, its highest atomic density among all solids, and its remarkably high thermal conductivity, while being an insulator. Recently, the RBS/C technique has been applied in studying the diamond amorphization induced by the ion implantation [17-19]. Moreover, artificial diamonds are widely used nowadays for the creation of state of the art radiation detectors [20]. A comprehensive study of the dechanneling of $\mathrm{MeV}$ protons in diamond crystals showed that the experimentally estimated dechanneling profile had a shape of a sigmoidal dechanneling function [21]. In the eighties, Fearick and Sellschop investigated the energy loss of protons for the main crystallographic axes of diamond in the transmission geometry [22]. They obtained the ratio between the channeling to random energy loss for a crystal thickness of $18.7 \mu \mathrm{m}$ and 2 to $12 \mathrm{MeV}$ protons. However, there is a remarkable lack in the literature concerning backscattering studies of $\mathrm{MeV}$ protons on diamond crystals. 
The present article investigates experimentally and via a new computer code, EBS/C spectra of protons, in the energy range from $1.0 \mathrm{MeV}$ to $1.9 \mathrm{MeV}$ for the $<100>$ diamond crystal orientation. In this energy range, proton elastic scattering on carbon exhibits a distinct strong resonance at $1737 \mathrm{keV}$ [23]. The existence of a resonance in elastic scattering acts as an essential energy marker, providing steep maxima and minima in the experimental yield, which can be subsequently used to unambiguously validate the quality of the computer simulation and the energy trend of the implemented parameters.

\section{Experimental setup}

The experiment was conducted at Ruđer Bošković Institute, Zagreb, Croatia, using the $1 \mathrm{MV}$ HV Tandetron accelerator, which is capable of delivering protons in the 0.5 to 2.0 $\mathrm{MeV}$ range, with variable energy steps. The beam line on which the goniometer was installed is designed in such a way, as to minimize the angular divergence of the incoming ion beam, by avoiding the use of a focalizing quadrupole magnet. The collimator system consisted of two small apertures, $1 \mathrm{~mm}$ in diameter, placed at 12 and $37 \mathrm{~cm}$ from the target holder. The goniometer allowed for a five-degrees of freedom movement, as well as for the positioning of the targets with an angular accuracy of $0.01^{0}$. The beam spot size was 0.8 $\mathrm{mm}^{2}$. A Silicon surface barrier detector (SSB), set at $160^{\circ}$, having a thickness of $300 \mu \mathrm{m}$, was used for the recording of the experimental EBS/C spectra. The targets used were artificial diamond crystals of about $3 \times 3 \mathrm{~mm}^{2}$ in size, produced by the "Element 6" company. They were cut perpendicularly to their $<100>$ axis. The density of the diamond crystals was the nominal one, namely $\sim 3.51 \mathrm{~g} / \mathrm{cm}^{3}$.

The diamond crystal was aligned using the $1 \mathrm{MeV}$ proton beam. In order to reduce any possible radiation damage, the current on the target never exceeded $4 \mathrm{nA}$, while for the same reason, all EBS/C spectra were acquired using a minimal total dose, though adequate to yield sufficient statistics. After the correct positioning of the beam spot on the diamond, the angular scans (tilt, azimuth) were initially performed. Fig. 1 shows a channeling/blocking pattern of a diamond crystal obtained after an angular scan. The maximum number of steps, around 30 for both angles, corresponds to $\sim 3^{0}$. The center of the channel seen in Fig. 1 denotes the smallest yield, whose values for tilt and azimuth were taken as the optimal $<100>$ channeling direction, and henceforth used for acquiring the EBS/C spectra. The random crystal orientation was determined using the standard technique of tilting the sample by several degrees relative to the channeling direction, and was verified via further angular scans. EBS/C spectra were acquired in the random and in the $<100>$ channeling orientations for $1.0 \mathrm{MeV}, 1.2 \mathrm{MeV}, 1.5 \mathrm{MeV}, 1.8 \mathrm{MeV}, 1.825 \mathrm{MeV}, 1.85$ $\mathrm{MeV}, 1.875 \mathrm{MeV}$ and $1.9 \mathrm{MeV}$ incident proton beams. 


\section{Computer simulation code}

The computer program code CSIM (Channeling SIMulations) applied was developed at the Laboratory of Physics, Vinča Institute of Nuclear Sciences. The beta version of the code will soon be available to the scientific community for downloading and testing via the Institute's web site. It simulates experimentally obtained EBS spectra, both random and channeling ones, and has substantial improvements from the computer program presented in the work of Aslanoglou et al. [12]. The program takes as input values the atom types of which the crystal is made, with the option of using manually set density values (as in this particular case), instead of standard ones (determined by using the Bragg's rule). The target in the program can be divided into computational blocks of adjustable, variable thickness, but in the present case it was set at $40 \mathrm{~nm}$, the choice of which proved to be the optimal one, taking into account two opposing requirements: the accuracy of the obtained backscattering spectra and the computing time. It was written using the $\mathrm{C}++$ object oriented language.

In the case of the random orientation, after the ion beam enters a specific computational block, the probability for backscattering is computed via a linear interpolation of the cross section obtained from the evaluated differential cross section data from SigmaCalc 2.0 by using the .r33 file format in the selected energy range, for the selected scattering angle [23], or by adopting the Rutherford formula when elastic backscattering differential cross section data is not available. The ion beam energy loss in a selected block is calculated using Ziegler and Biersack's stopping power data [24], while the energy straggling in each block is calculated as the sum of 1) the non-statistical energy broadening induced by the energy loss in the layer and 2) the statistical one originating from the different energies of the ions in the beam, calculated using Yang's empirical formula [25]:

$$
\sigma_{\text {out }}^{2}=\left(\frac{S_{\text {out }}}{s_{\text {in }}}\right)^{2} \sigma_{\text {in }}^{2}+\sigma_{\text {Yang }}^{2}
$$

where $\sigma_{\text {out }}$ and $\sigma_{\text {in }}$ are the ion beam energy widths, while $S_{\text {out }}$ and $S_{\text {in }}$ are the stopping powers at the end and at the beginning of each block, respectively, and $\sigma_{\text {Yang }}$ denotes the statistical energy broadening. The backscattered part of the ion beam is traced back to the detector through the previously propagated blocks, affected again by the energy loss and straggling, where a correction for the detector resolution is finally added to the ion beam straggling as:

$$
\sigma^{2}=\sigma_{\text {str }}^{2}+\sigma_{\text {det }}^{2}
$$

where $\sigma$ is the final energy width, $\sigma_{\text {str }}$ is the straggling that occurred inside the target and $\sigma_{\text {det }}$ is the detector resolution. The backscattered ion beam from each block is then 
represented in a Gaussian form, the sum of which makes the resulting spectrum. Upon adding the backscattered part of the ion beam in the spectrum, the ion beam propagation proceeds to the next computational block where calculations undertake all the above mentioned steps, till the ion beam energy falls below $100 \mathrm{keV}$. The final simulated spectrum is rebinned according to the experimental ADC calibration settings.

In the case of channeling orientation, the program assumes a Gompertz type sigmoidal dechanneling function given by $[15,16]$ :

$$
N_{d}=N_{0} \frac{e^{-e^{-k\left(x-x_{c}\right)}}-e^{-e^{k x_{c}}}}{1-e^{-e^{k x_{c}}}}
$$

where $N_{d}$ and $N_{0}$ represent the number of dechanneled and initial ions, respectively, $k$ is the dechanneling rate (slope at the inflection point of the sigmoidal function), $x$ is the crystal depth, and $x_{C}$ is the dechanneling range (the inflection point of the sigmoidal function). As a first order approximation, it is assumed that the initial number of dechanneled ions is zero, and that it approaches $N_{0}$ for very large crystal depths.

The channeling case is treated in the following way: In each block, the energy loss and energy straggling are calculated in the same way as in the random orientation with one significant difference, namely, they are multiplied by (an ab initio assumed constant over the whole distance the ion travels in the channeling mode inside the crystal) coefficient $\alpha$, which is defined as the ratio of the stopping power in the channeling to the stopping power in the random orientation. The backscattering of ions while being channeled has been neglected, since a perfect crystalline structure without impurities was assumed. The dechanneled part of the ion beam in each block has been evaluated by Eq. 3., and was further treated as being 'random', starting from that block with appropriate energy, whose further propagation into the crystal and subsequent backscattering contributes to the resulting EBS spectrum. The calculation then proceeds to the next computational block where it undertakes the aforementioned calculations. Therefore, the program takes approximately an order of magnitude more time computing EBS spectra in the channeling orientation than in the random one. Still, the calculation is very fast compared to MC codes based on the BCA approach. It should be mentioned here that our program, like e.g. SIMNRA, is a phenomenological one. It has been proven that this kind of programs dominate in practice in all ion beam analysis applications in which a reasonable computational time over the reliable quantification of data is required.

In order to properly describe the channeling EBS spectrum there are thus three fitting parameters: $\alpha$, the channeling to random stopping ratio, $k$ the dechanneling rate and $x_{C}$ the dechanneling range. It is possible for the user of the code, to set fixed values for these three parameters. However, if an experimental spectrum is provided, it is also possible to 
perform a $\chi^{2}$ minimization using the MINUIT minimization routine, developed at CERN, rewritten in $\mathrm{C}++[26]$ and embedded in the code.

\section{Results and discussion}

The experimental random and channeling elastic backscattering spectra were fitted by the described computer code for the proton beam energies of $1.0 \mathrm{MeV}, 1.2 \mathrm{MeV}, 1.5 \mathrm{MeV}$, 1.8 MeV, 1.825 MeV, 1.85 MeV, 1.875 MeV and 1.9 MeV. As it has been already mentioned, the choice of the energy range is closely related to the existence of a strong resonance of the ${ }^{12} \mathrm{C}\left(\mathrm{p}, \mathrm{p}_{0}\right){ }^{12} \mathrm{C}$ elastic scattering at $1737 \mathrm{keV}(\Gamma=47 \mathrm{keV})$ evident in EBS/C spectra. Also, the presence of a strong resonance structure in the experimental EBS/C spectra acts as a distinct energy marker, causing strong maxima and minima in the experimental yield, thus limiting the number of available parameter sets $\left(\alpha, k, x_{C}\right)$ to which the $\chi^{2}$ minimization routine can converge. The random spectra were used as reference points for the energy calibration and the eventual fine tuning of the ion beam energy which presented an initial offset of 2-7 keV. The energy ripple on the other hand did not exceed $0.1 \%$ (as verified in previous runs).

The ${ }^{12} \mathrm{C}\left(\mathrm{p}, \mathrm{p}_{0}\right)^{12} \mathrm{C}$ elastic scattering differential cross sections for the detector angle position of $160^{\circ}$ used in the EBS/C computer simulations were taken from SigmaCalc 2.0 via the IBANDL online library [23]. Fig. 2 depicts the measured spectrum for $1.8 \mathrm{MeV}$ protons in the random orientation, as well as, its computer simulated fitting curves with our code and the commonly used SIMNRA package [27]. The quality of the code in comparison with SIMNRA is excellent, with the difference between the two being less than $1 \%$. For the incident proton energy of $1.8 \mathrm{MeV}$, one can clearly observe the distinct resonance structure at $1737 \mathrm{keV}$. In the case of the random orientation the peak owing to its close proximity to the surface, is obviously much more pronounced than in the channeling one (see Fig 3a), where only a small number of incoming protons have been dechanneled and subsequently backscattered, thus contributing to the occurring spectrum. As shown in Fig. 2, both computer simulated spectra reproduce the experimental ones very well.

Figs. 3(a)-(e) show experimental spectra along with our computer simulated ones for the proton beam energies of $1.8 \mathrm{MeV}, 1.825 \mathrm{MeV}, 1.85 \mathrm{MeV}, 1.875 \mathrm{MeV}$ and $1.9 \mathrm{MeV}$, in the channeling $<100>$ diamond crystal direction. The analysis shows that the $\chi^{2}$ minimization routine converges to a unique solution due to the distinct resonance peak in ${ }^{12} \mathrm{C}\left(\mathrm{p}, \mathrm{p}_{0}\right){ }^{12} \mathrm{C}$ reaction at $1737 \mathrm{keV}$, with its pronounced minima and maxima, obtaining a very good agreement between simulated and experimental EBS/C spectra in all studied cases, as shown in Figs. 3(a)-(e). Additionally, this justifies the implementation of a Gompertz type sigmoidal dechanneling function. The reproduction of random spectra was also very good 
in all studied cases. It should be noted here that the crystal surface effects (e.g. the surface peak) are not currently treated by the code.

The variation of the stopping power ratio $\alpha$ with respect to the beam energy in the 1.8 $\mathrm{MeV}-1.9 \mathrm{MeV}$ energy range, as obtained via the fitting procedure, is presented in Fig. 4. One can observe its rather weak dependence on ion beam energy, with values in the 0.860.90 range and an average value of 0.88 . This leads to the conclusion of a relative invariance of $\alpha$ with respect to the ion beam energy. It was determined in the past that $\alpha$ values for the $<100>$ silicon channel assume values closer to 0.7 [12], and indeed silicon is a diamond-cubic type crystal. However, the distance between carbon atoms in the nods of a diamond lattice is smaller than in the case of silicon, namely $0.356 \mathrm{~nm}$ versus $0.543 \mathrm{~nm}$, respectively, leading to a higher electron density in the diamond channel and thus to a more pronounced energy loss, hence to higher values for $\alpha$, despite the excellent crystalline quality of the irradiated artificial diamond targets.

As it has already been mentioned, having a distinct resonance peak in ${ }^{12} \mathrm{C}\left(\mathrm{p}, \mathrm{p}_{0}\right)^{12} \mathrm{C}$ at $1737 \mathrm{keV}$ leads the $\chi^{2}$ minimization routine to a unique solution and a very good agreement between simulated and experimental EBS/C spectra. However, for the $1.0 \mathrm{MeV}, 1.2 \mathrm{MeV}$ and $1.5 \mathrm{MeV}$ proton backscattering spectra there is no distinct resonant peak. As a result, the analysis shows that multiple sets of the fitting channeling parameters $\left(\alpha, k, x_{C}\right)$ through the $\chi^{2}$ minimization process can occur, all demonstrating a good agreement between the simulated and the experimental EBS/C spectra. Because of this, for the proton energy range of $1.0 \mathrm{MeV}-1.5 \mathrm{MeV}$, the stopping power ratio, $\alpha$, was set and kept fixed to its average value of 0.88 while $k$ and $x_{C}$ parameters were varied in the fitting procedure. Figs. 5(a) - (c) show the experimental and simulated EBS/C spectra for the proton beam energies of 1.0 $\mathrm{MeV}, 1.2 \mathrm{MeV}$ and $1.5 \mathrm{MeV}$, respectively. It is clear that the agreement between the spectra is very good. Nonetheless, it is also mathematically evident that the determination of $k$ and $x_{C}$ through the $\chi^{2}$ minimization in this case cannot be unambiguous, due to the lack of pronounced maxima and minima in the experimental spectra. Several combinations of $k$ and $x_{C}$ can lead to the same high-quality reproduction of EBS/C spectra and they can be, either determined by using the fitting routine of the code, or manually set by the user. It should also be noted that in all the cases considered here the $\chi^{2}$ minimization routine did not take into account parts of spectra corresponding to energies below $300 \mathrm{keV}$ due to the large energy loss straggling uncertainty.

Figs. 6 and 7 show dependencies of the dechanneling rate and range on the ion beam energy respectively, when the resonance is present in the experimental channeling spectra. From Fig. 6 one can observe the general trend of declining of parameter $k$ with ion beam energy, which is in accordance to its physical interpretation. The slowing down of the dechanneling process with increasing ion beam energy is expected, since the 'same' number of ion-electron scattering events, which constitutes the main ion dechanneling 
mechanism, occurs over greater crystal lengths for higher ion beam energies than for lower ones. In Fig. 7, for the dependence of the dechanneling range, $x_{C}$, on the ion beam energy, one can observe the general trend of its increase, which is also in accordance with its physical interpretation as corresponding to the dechanneling range.

Additionally, it should be stressed here that the new CSIM code offers clear improvements and advantages over the one presented in the past [15]. Namely, the possibility to use evaluated differential cross section files from IBANDL, or recommended, experimentally determined ones in the .r33 format, the correct treatment of the energy straggling effect via widely used models, the ability to modify the implemented stopping power values and the density of each target. Moreover, its modular structure allows for the future treatment of surface effects and the study of deep implanted ion concentration profiles, either following a random or a channeling implantation. Also, in our opinion, the most important result using the CSIM code is that, since the differential cross sections are no longer obtained from the deconvolution of thick target yields, as in [15], the Gompertz dechanneling parameters $k$ and $x_{C}$ are both qualitatively following the correct energy trend, as predicted by the MC calculations based on the BCA approach. As it was already mentioned, the possibility to analyze EBS/C spectra in the presence of resonances, which provide the user with well-defined energy markers, leads to a superior reproduction of the experimental channeling spectra and to an unambiguous determination of the corresponding channeling parameters, which is not possible when smooth RBS/C spectra are involved.

\section{Conclusions}

In the present work experimentally obtained random and channeling backscattering spectra from an artificial virgin diamond crystal for the beam energies from $1.0 \mathrm{MeV}$ to 1.9 $\mathrm{MeV}$, with a dominant resonance at $1737 \mathrm{keV}$ in proton elastic scattering on carbon, were successfully fitted for the first time using a new computer code, CSIM. The program assumed a Gompertz type sigmoidal dechanneling function with two parameters, $k$ and $x_{C}$, corresponding to the dechanneling rate and range, respectively. The energy dependence of both parameters, decreasing for $k$ and increasing for $x_{C}$, is in accordance with their perceived physical interpretation. Further, the program uses $\alpha$, ratio of the channeling to random energy losses, as a fitting parameter. For the beam energy range of $1.8 \mathrm{MeV}-1.9$ $\mathrm{MeV}$, when steep maxima and minima in the experimental yields occur due to the resonant proton-carbon scattering, the fitting procedure results in unique solutions for the channeling parameters $\left(\alpha, k, x_{C}\right)$. For the non-resonant cases under consideration, namely for the proton beams of $1.0 \mathrm{MeV}, 1.2 \mathrm{MeV}$ and $1.5 \mathrm{MeV}$, a unique solution of the channeling parameters was not possible due to the rather smooth shape of the experimental spectra, 
and thus a fixed value of the parameter $\alpha$, being the average value for the resonant cases, was assumed, in order to maintain the quality of the simulation, while simultaneously complying with the physical boundaries and the correct energy trend of the implemented parameters.

EBS/C as a technique in Ion Beam Analysis (IBA) offers the possibility for determining concentration profiles of light elements implanted or generally present as interstitial impurities in heavier crystalline matrices, or for the detection of heavier elements in higher depths, whose yield in EBS spectra overlaps with the matrix-induced background. The advantage of using EBS/C in such cases lies in the fact that NRA differential cross sections are at least one or more orders of magnitude lower than elastic scattering ones, thus the LLD levels for the determination of impurities are significantly enhanced. However, there are still quite a few problems that need to be solved in order to successfully replace NRA with EBS/C in such cases, namely the comprehensive understanding of the dechanneling process, the treatment of surface distortion effects, the treatment of additional backscattering on impurities even when an ion is being channeled and the introduction of an amorphization profile, often induced by ion implantation or other mechanical stress, which also induces additional backscattering by matrix atoms. Our future research will be concentrated along these lines.

\section{Acknowledgements}

ME and SP acknowledge the support by the Ministry of Education, Science and Technological Development of Serbia through project "Physics and Chemistry with Ion Beams", No. III45006. ME, SP and MK acknowledge that this work is part of the NTUA//VINS Collaboration Agreement. MK and SP acknowledge partial support by the Horizon 2020 project AIDA-2020, GA No. 654168. SF acknowledges the support of the Croatian Science Foundation under the project MIOBICC (No. 8127). 


\section{References}

[1] D. Gemmell, Rev. Mod. Phys., pp. 46, 129, 1974.

[2] G. Foti, F. Grasso, R. Quattrochi and E. Rimini, Phys. Rev. B, vol. 3, p. 2169, 1971.

[3] J. Davies, L. Howe, D. Marsden and J. Whitton, Rad. Eff., vol. 12, p. 247, 1972.

[4] A. Fontell, E. Arminen and E. Leminen, Rad. Eff., vol. 12, p. 255, 1972.

[5] R. Hellborg, Phys. Scripta, vol. 4, p. 75, 1971.

[6] K. Bjorkqvist, B. Cartling and B. Domeij, Rad. Eff., vol. 12, p. 267, 1972.

[7] T. Oshiyama and M. Mannami, Phys. Lett. A, vol. 81, p. 43, 1981.

[8] V. Beloshitsky, F. Komarov and M. Kumakhov, Phys. Rep., vol. 139, p. 293, 1986.

[9] G. Lulli, E. Albertazzi, M. Bianconi, G. Bentini, R. Nipoti and R. Lotti, Nucl. Instr. and Meth. B, vol. 170, p. 1, 2000.

[10] A. Turos, L. Nowicki, A. Stonert, K. Pagowska, J. Jagielski and A. Mueklich, Nucl. Instr. Meth. B, vol. 268, p. 1718, 2010.

[11] E. Kotai, Nucl. Instr. and Meth. B, vol. 118, p. 43, 1996.

[12] X. Aslanoglou, P. Assimakopoulos, M. Kokkoris and E. Kossionides, Nucl. Instr. and Meth. B, vol. 140, p. 294, 1998.

[13] M. Robinson and I. Torrens, Phys. Rev. B, vol. 9, p. 5008, 1974.

[14] S. Shafiei and M. Lamechi-Rachti, Nucl. Instr. and Meth. B, vol. 366, p. 57, 2016.

[15] M. Kokkoris, G. Perdikakis, S. Kossionides, S. Petrović and E. Simoen, Euro. Phys. J. B, vol. 34, p. 257, 2003.

[16] S. Petrović, M. Erić, M. Kokkoris and N. Nešković, Nucl. Instr. and Meth. B, vol. 256, p. 177, 2007.

[17] M. Ogura, M. Hasegawa, Y. Tanaka and N. Kobayashi, Nuclr. Instr. and Meth. B, vol. 161, p. $1043,2000$. 
[18] E. Friedland, H. Carstanjen, G. Myburg and M. Nasr, Nucl. Instr. and Meth. B, vol. 230, p. $129,2005$.

[19] V. Drumm, A. Alves, B. Fairchild, K. Ganesan, J. McCallum, D. Jamieson, S. Prawer, S. Rubinov, R. Kalish and L. Feldman, Appl. Phys. Lett., vol. 98, p. 231904, 2011.

[20] J. Pietraszko, L. Fabbietti, W. Koenig and M. Weber, Nucl. Instr. and Meth. A, vol. 618, p. $121,2010$.

[21] R. Fearick, T. Derry and J. Sellschop, Phys. Rev. B, vol. 40, p. 4289, 1989.

[22] R. W. Fearick and J. P. F. Sellschop, Nucl. Instr. and Meth., vol. 168, p. 51, 1980.

[23] A. Gurbich, "Ion Beam Analysis Nuclear Data Library," [Online]. Available: https://www-nds.iaea.org/exfor/ibandl.htm.

[24] J. Ziegler, J. Biersack and U. Littmark, The Stopping and Range of Ions in Solids vol. 1, New York: Pergamon Press Inc., 1985.

[25] Q. Yang, D. O'Connor and Z. Wang, Nucl. Instr. and Meth. B, vol. 61, p. 149, 1991.

[26] F. James and M. Roos, Comp. Phys. Comm., vol. 10, p. 343, 1975.

[27] M. Mayer, "SIMNRA ver. 6.04," 1997. [Online]. Available: http://www.rzg.mpg.de/ mam/. 


\section{Figure captions:}

Fig. 1. Graph showing the alignment of $<100>$ diamond crystal channel via azimuth and tilt angle scan. The square $<100>$ channel is clearly visible.

Fig. 2. Backscattering spectra for $1.8 \mathrm{MeV}$ protons impinging ona randomly oriented diamond crystal, the corresponding computer simulated spectra and the SIMNRA simulated spectra.

Fig. 3. Experimental EBS/C spectra and the corresponding simulated ones for a) $1.8 \mathrm{MeV}$, b) $1.825 \mathrm{MeV}$, c) $1.85 \mathrm{MeV}$, d) $1.875 \mathrm{MeV}$ and e) $1.9 \mathrm{MeV}$ protons.

Fig. 4. The channeling to random stopping power ratio, $\alpha$, as a function of energy in the 1.8 $-1.9 \mathrm{MeV}$ energy range.

Fig. 5. Experimental EBS/C spectra and the corresponding simulated ones for a) $1.0 \mathrm{MeV}$, b) $1.2 \mathrm{MeV}$ and c) $1.5 \mathrm{MeV}$ protons.

Fig. 6. Dechanneling rate, $k$, as a function of proton energy in the $1.8-1.9 \mathrm{MeV}$ energy range.

Fig. 7. Dechanneling range, $x_{C}$, as a function of proton energy in the $1.8-1.9 \mathrm{MeV}$ energy range. 


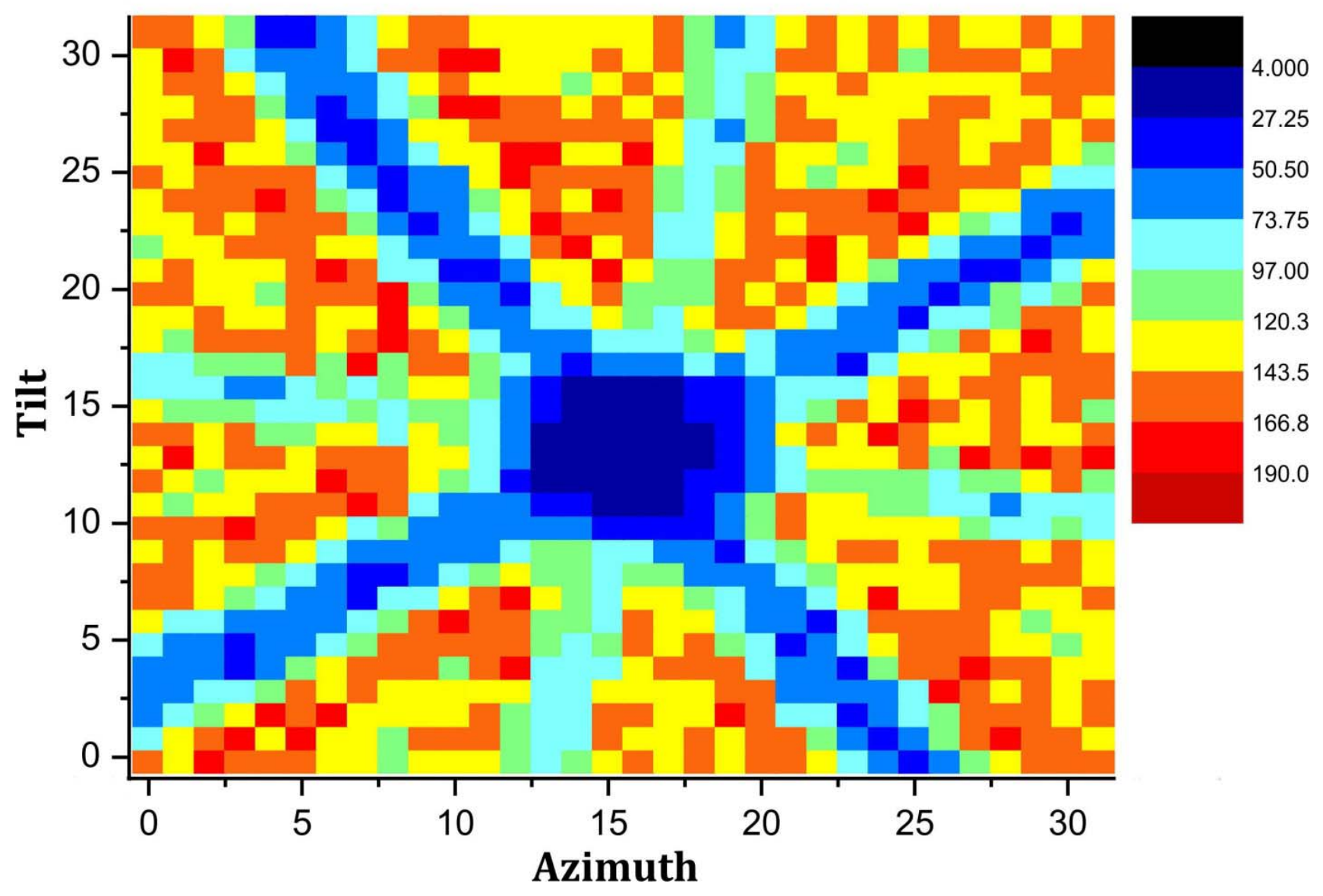

Fig. 1 


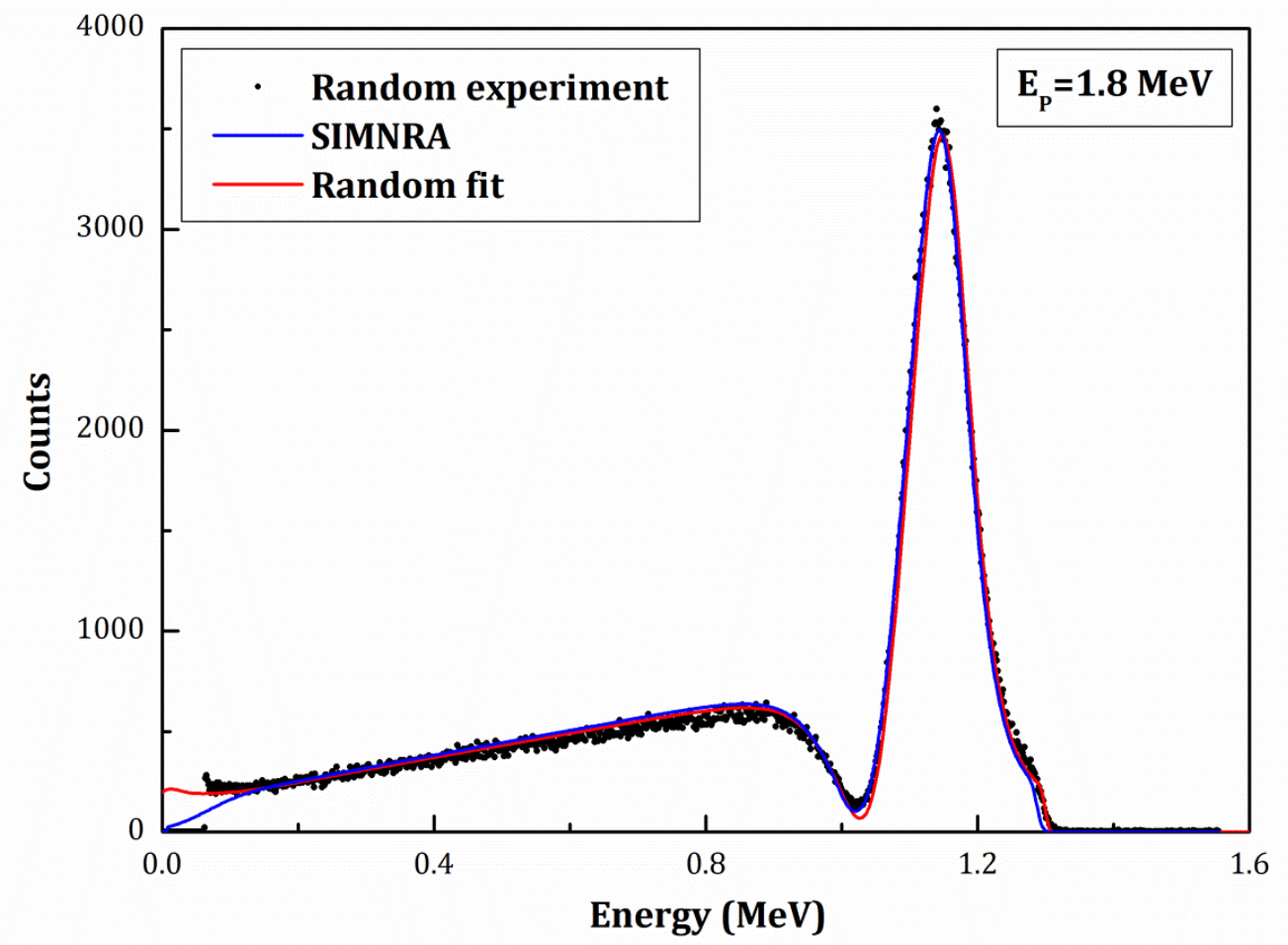

Fig. 2 


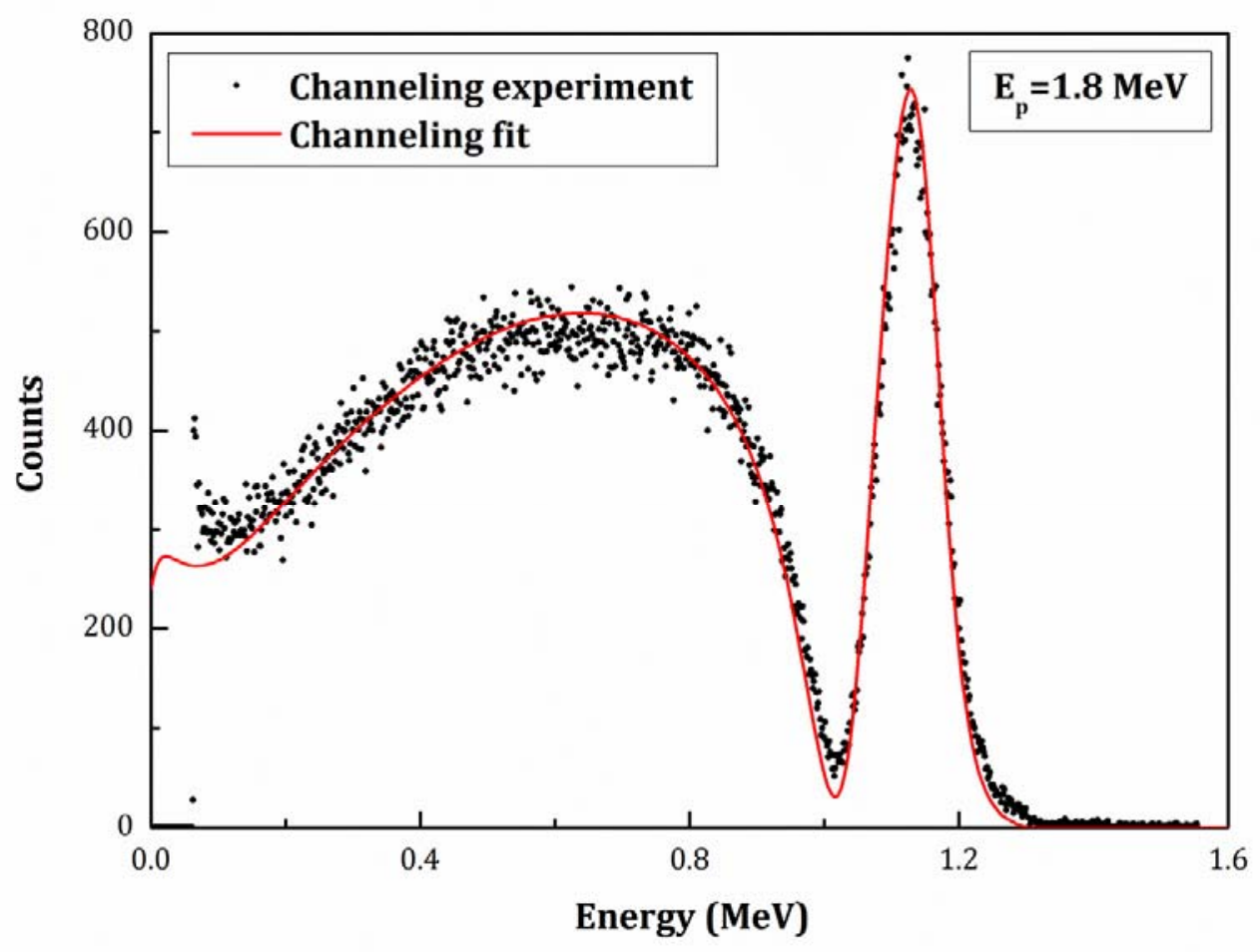

Fig. 3a 


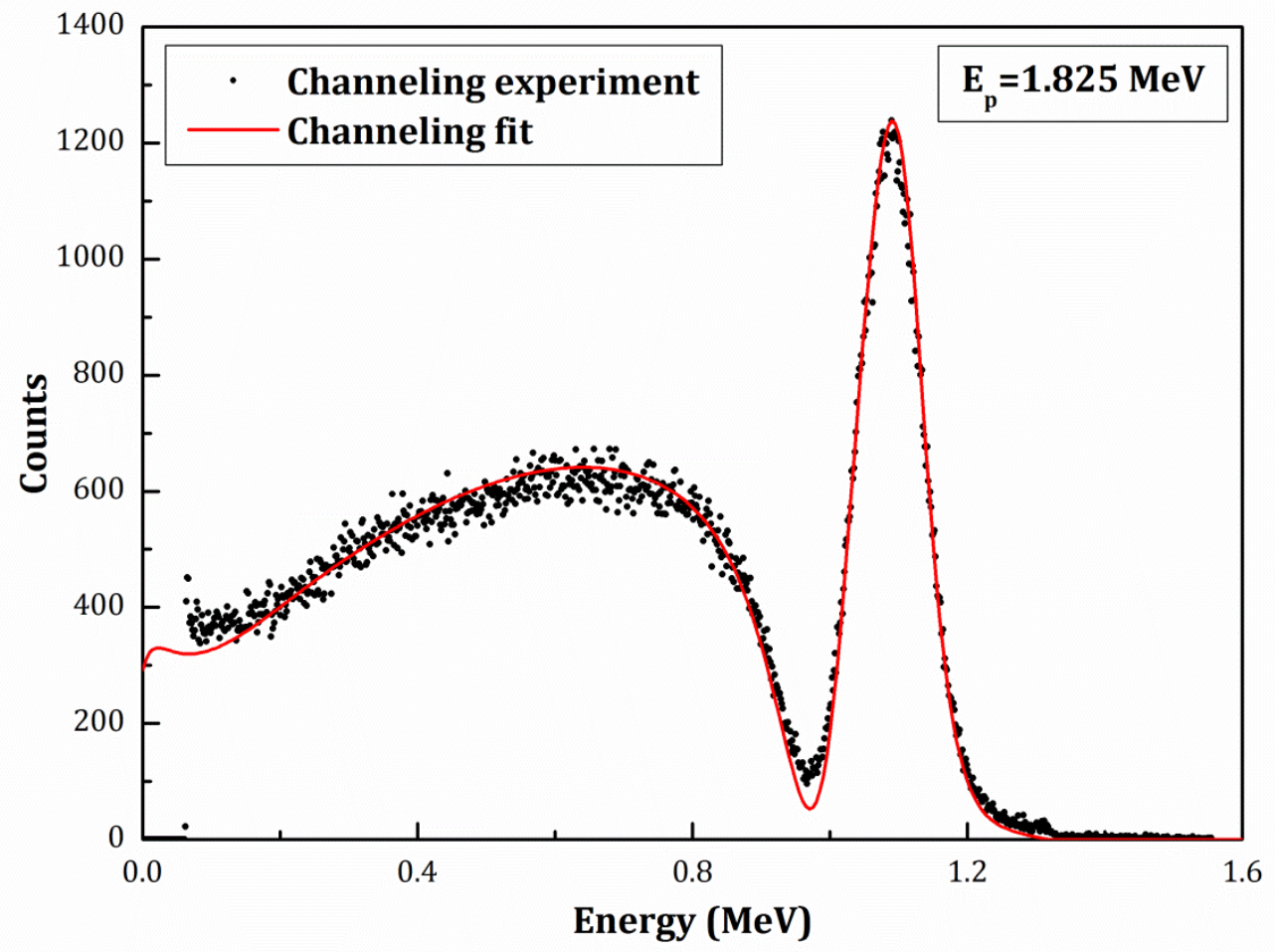

Fig. 3b 


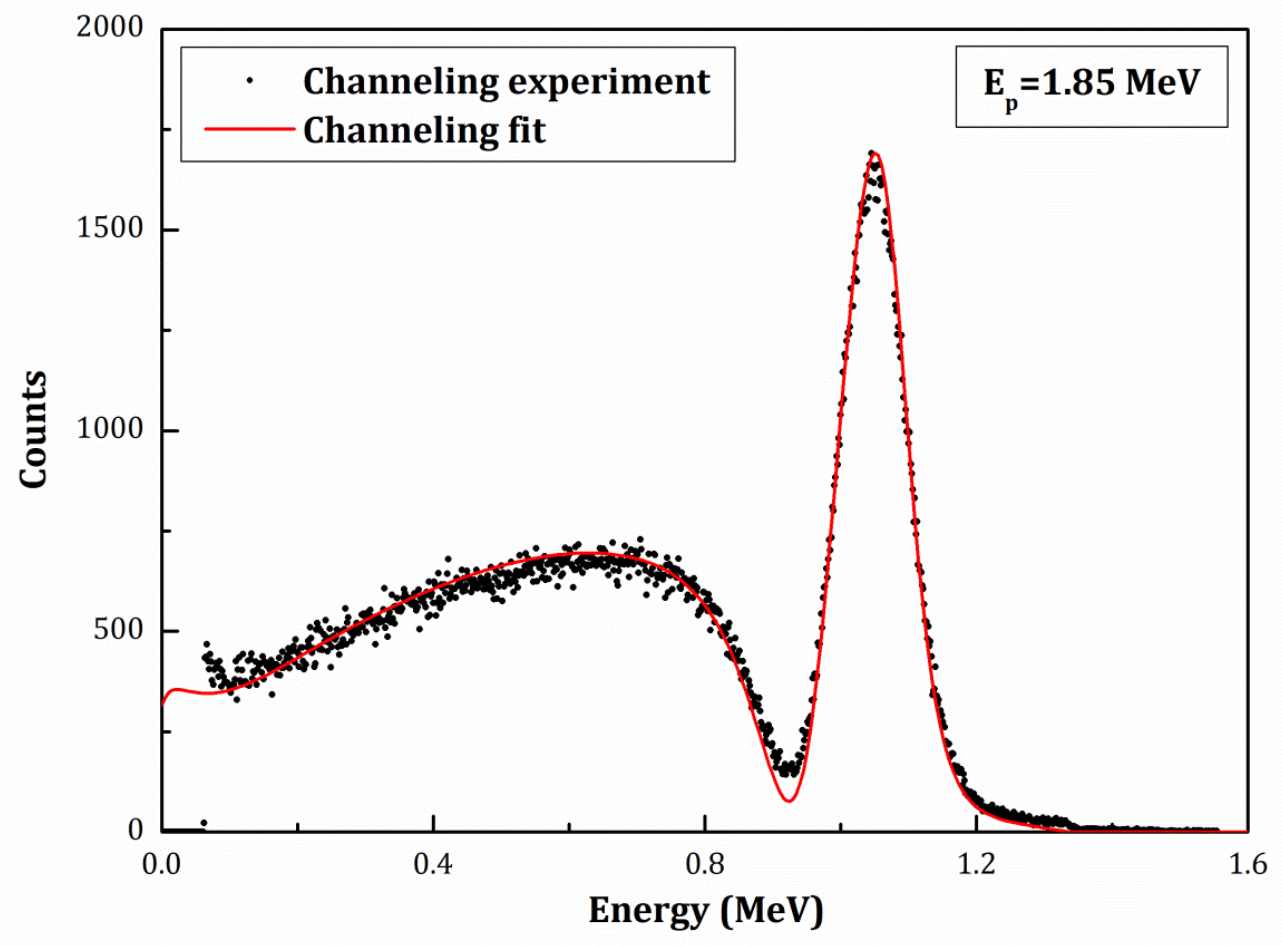

Fig. 3c 


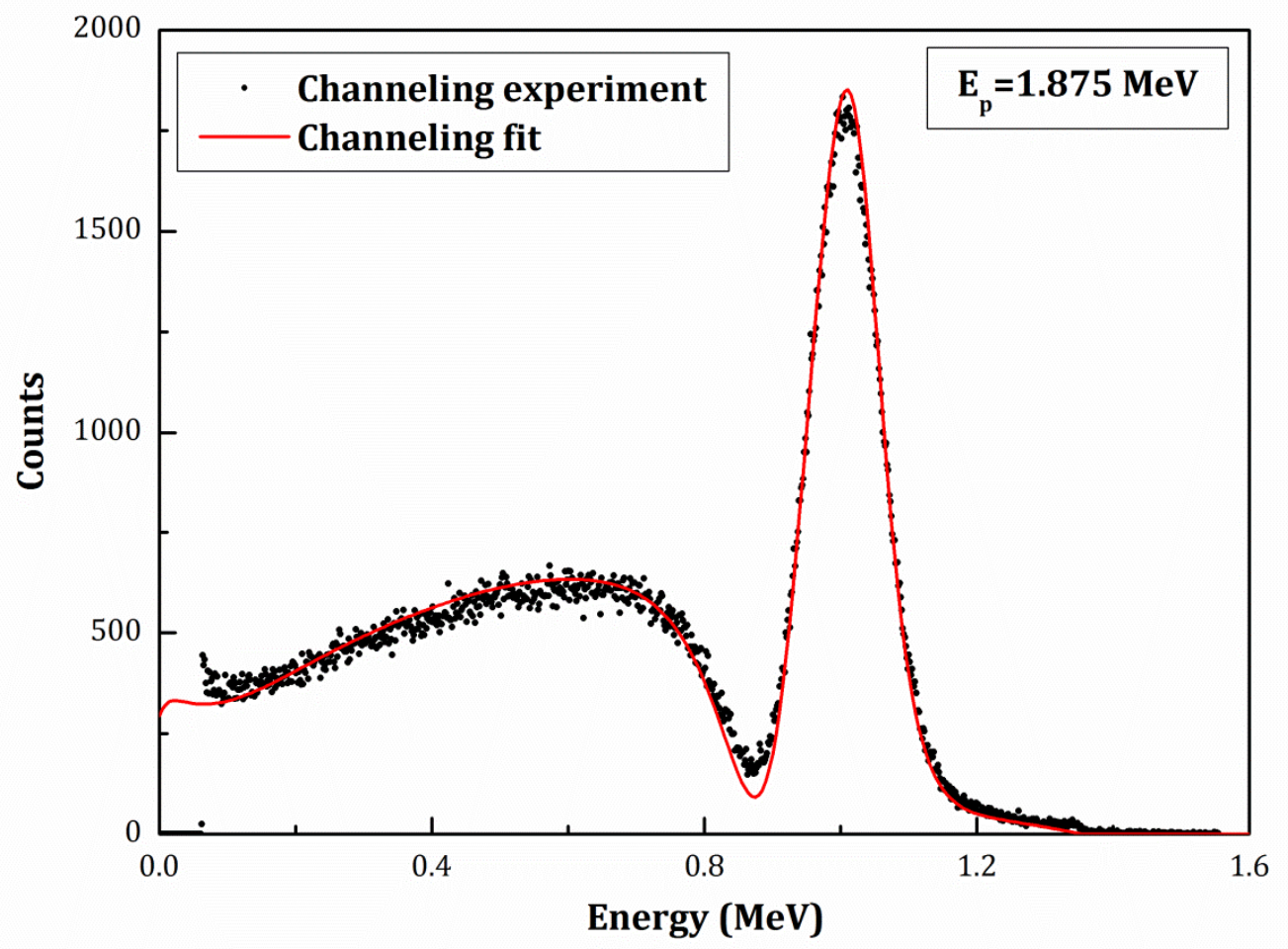

Fig. 3d 


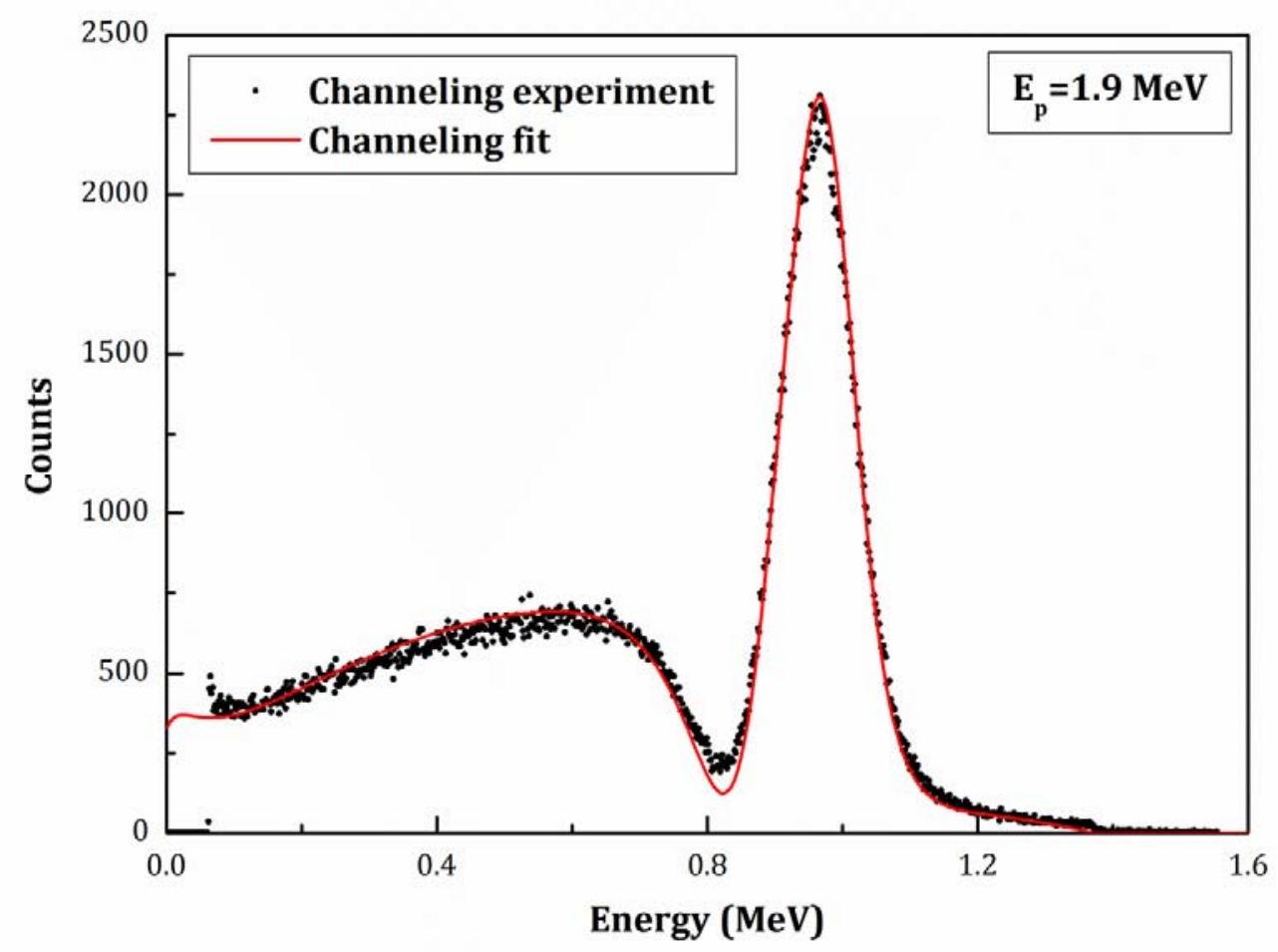

Fig. 3e 


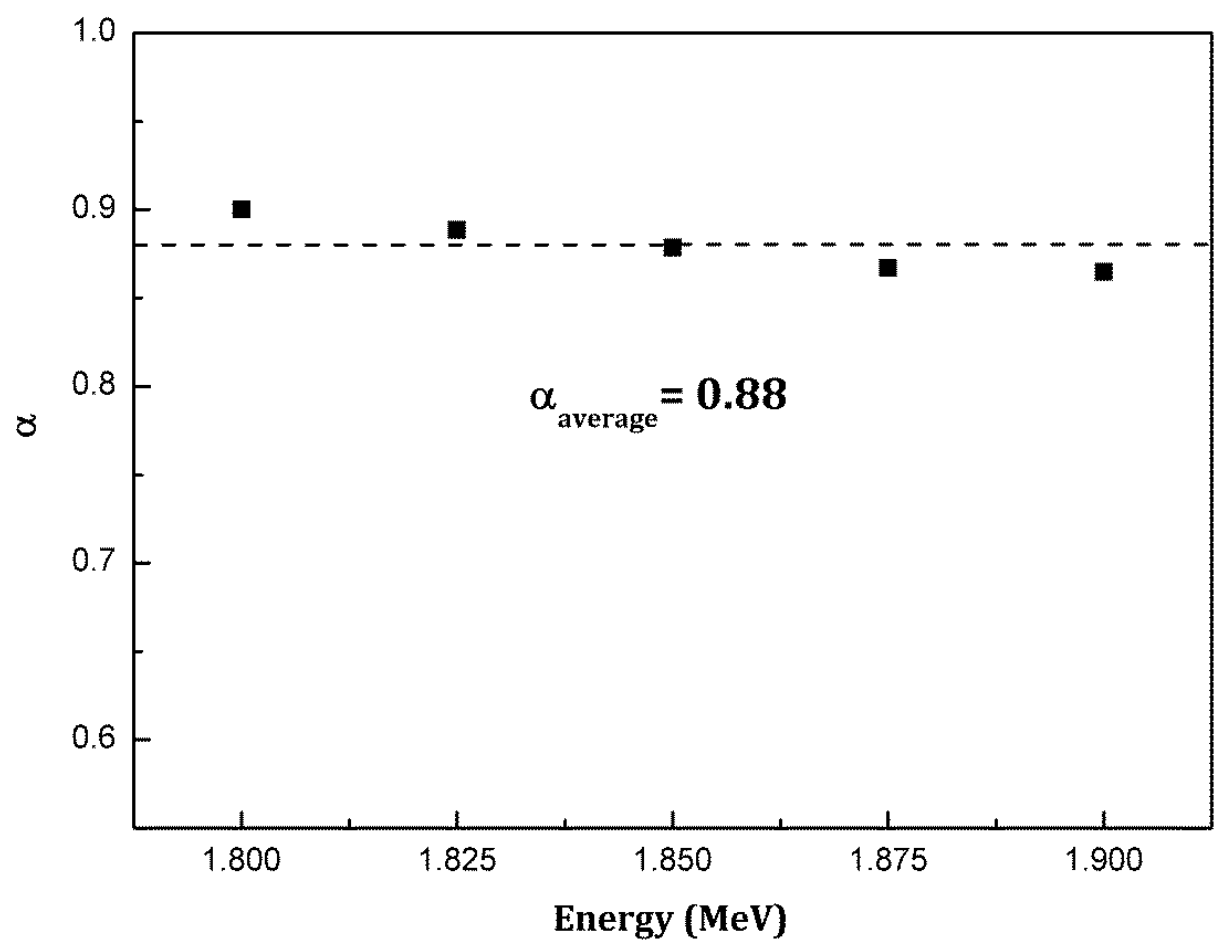

Fig. 4 


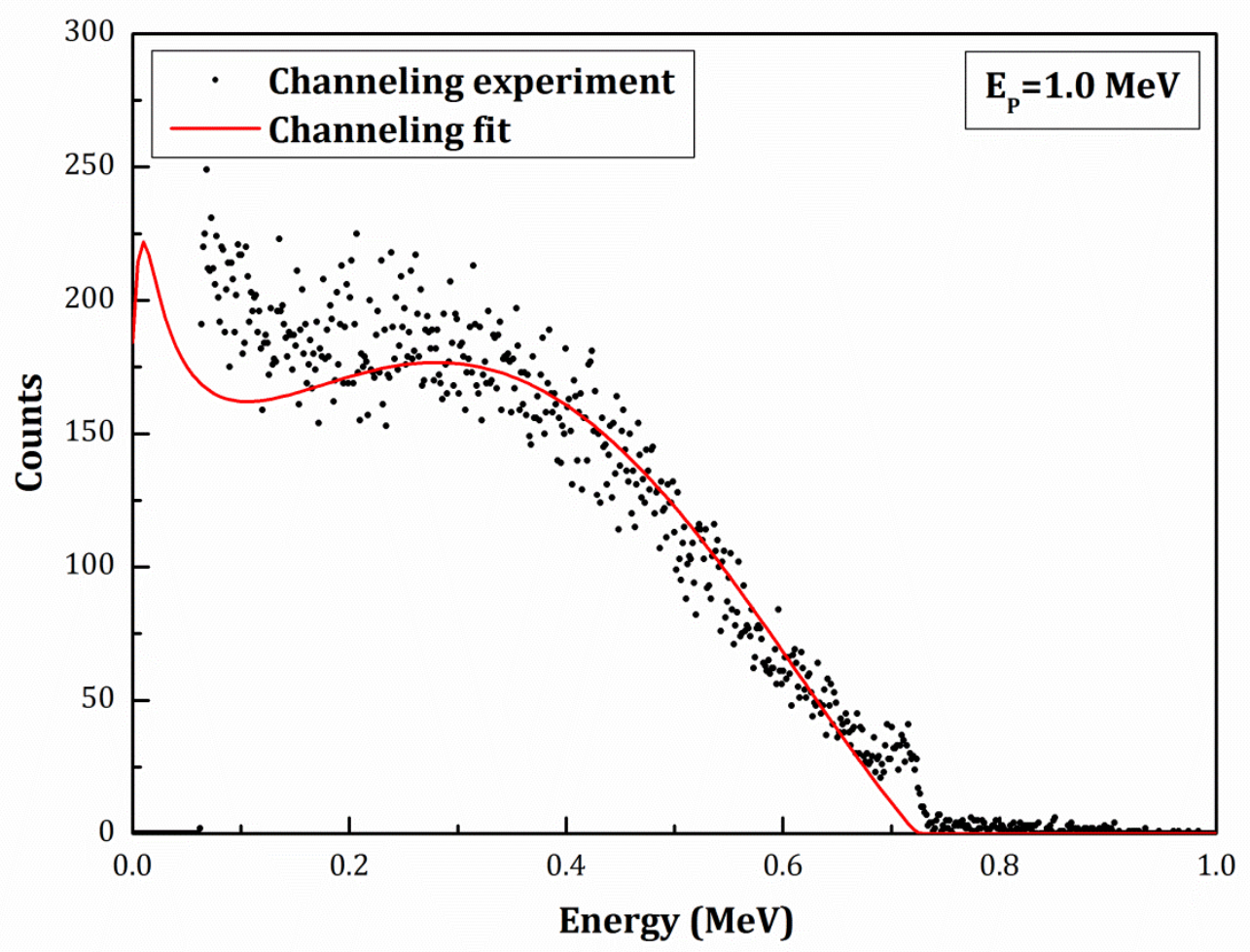

Fig. 5a 


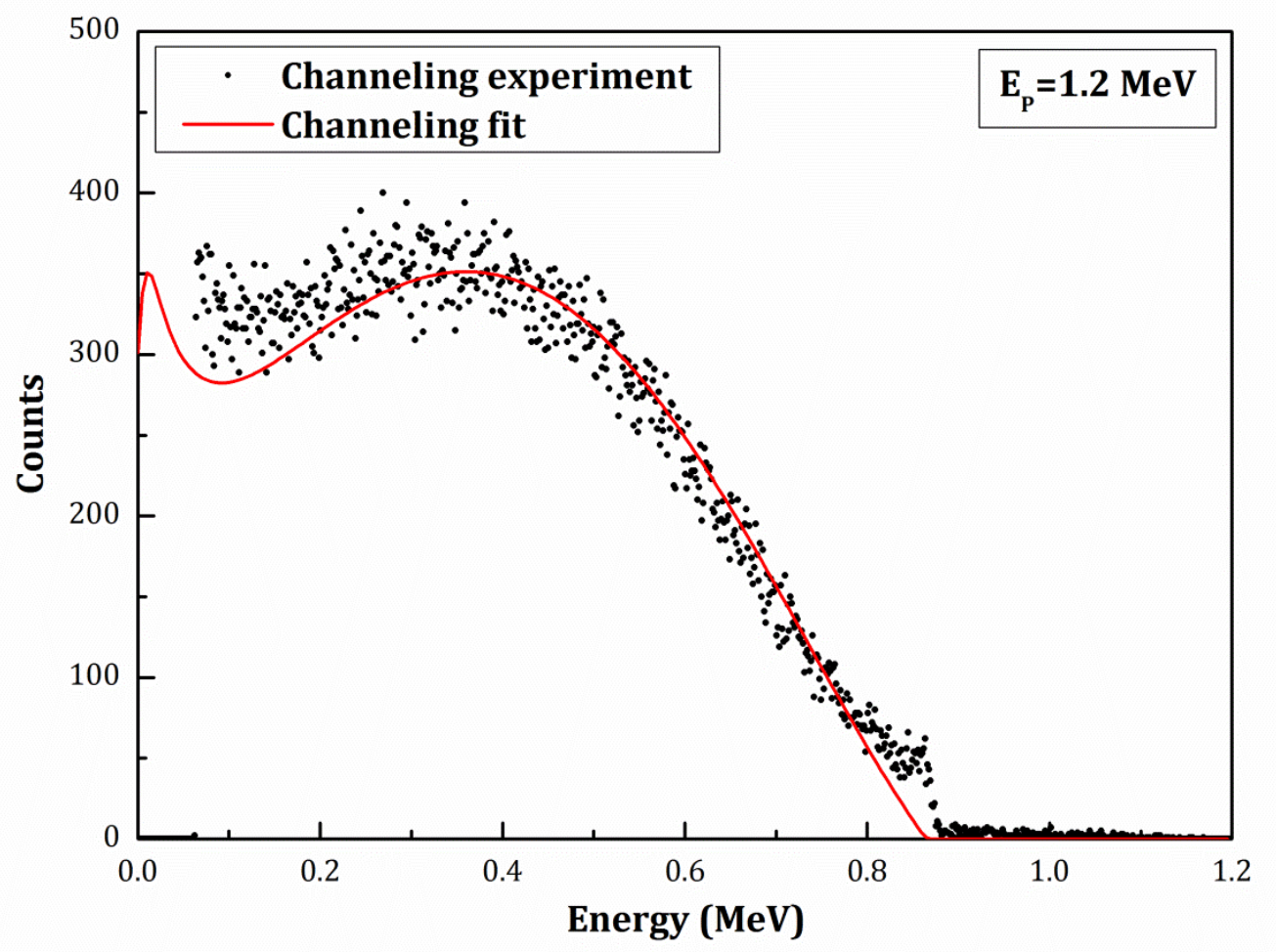

Fig. 5b 


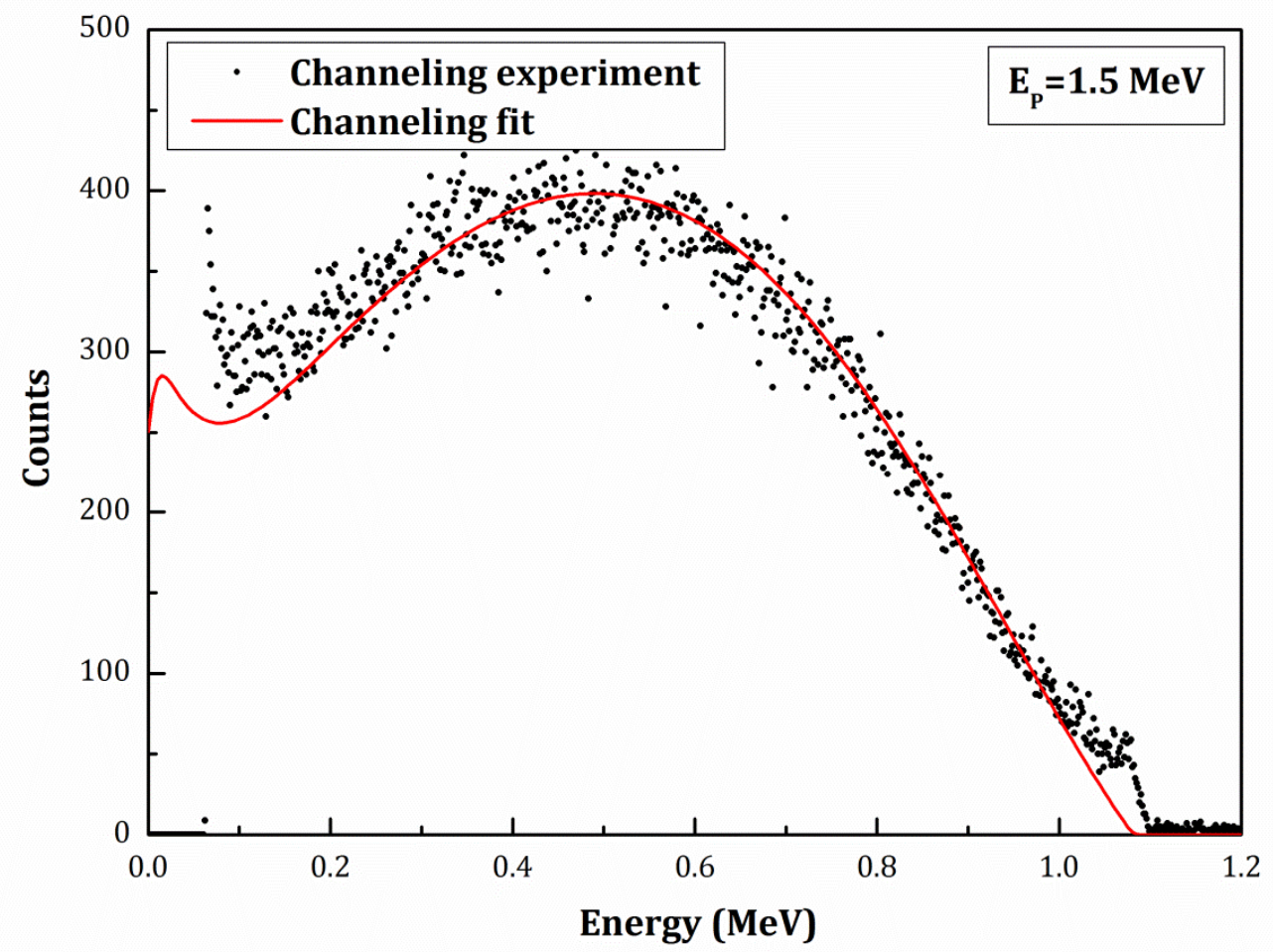

Fig. 5c 


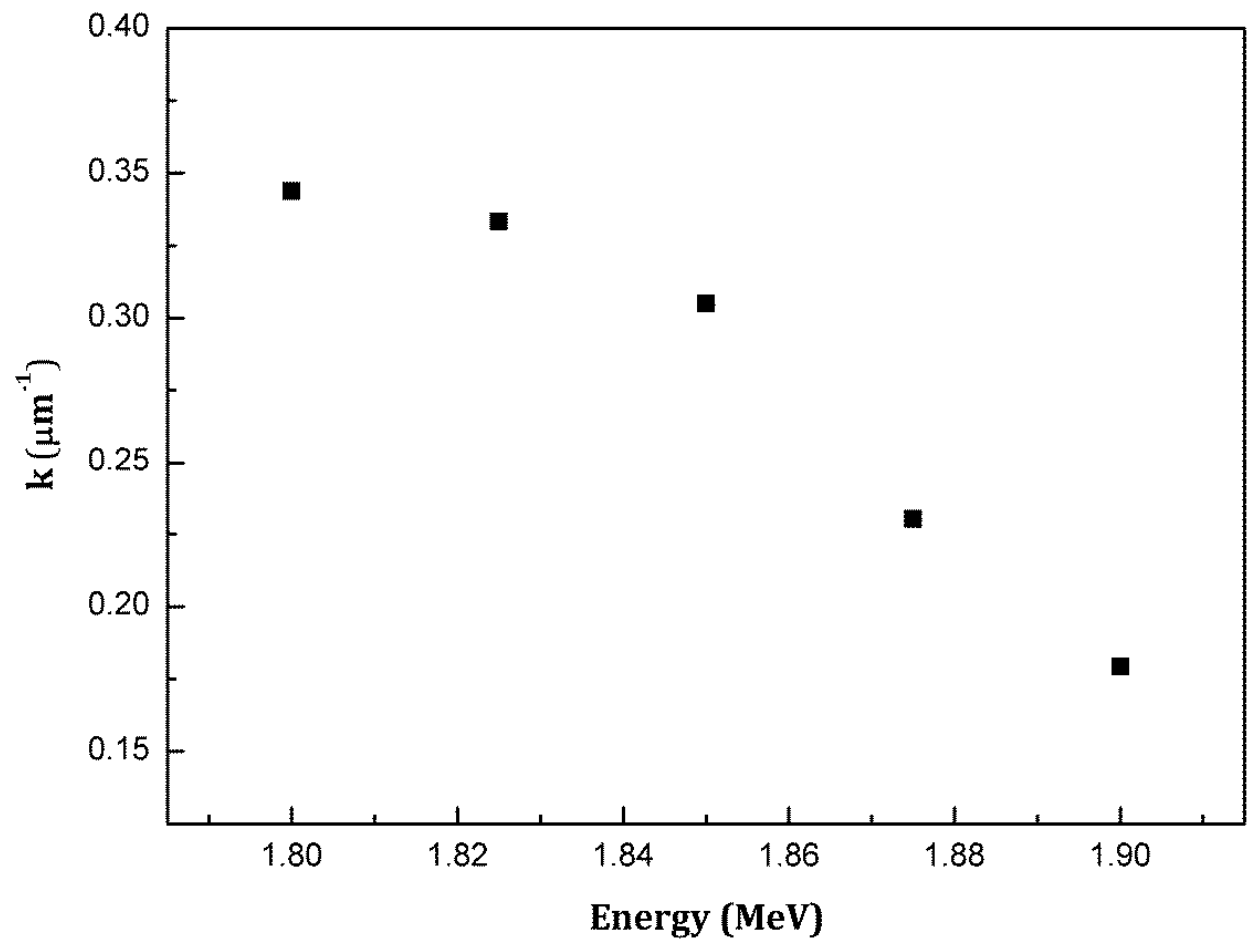

Fig. 6 


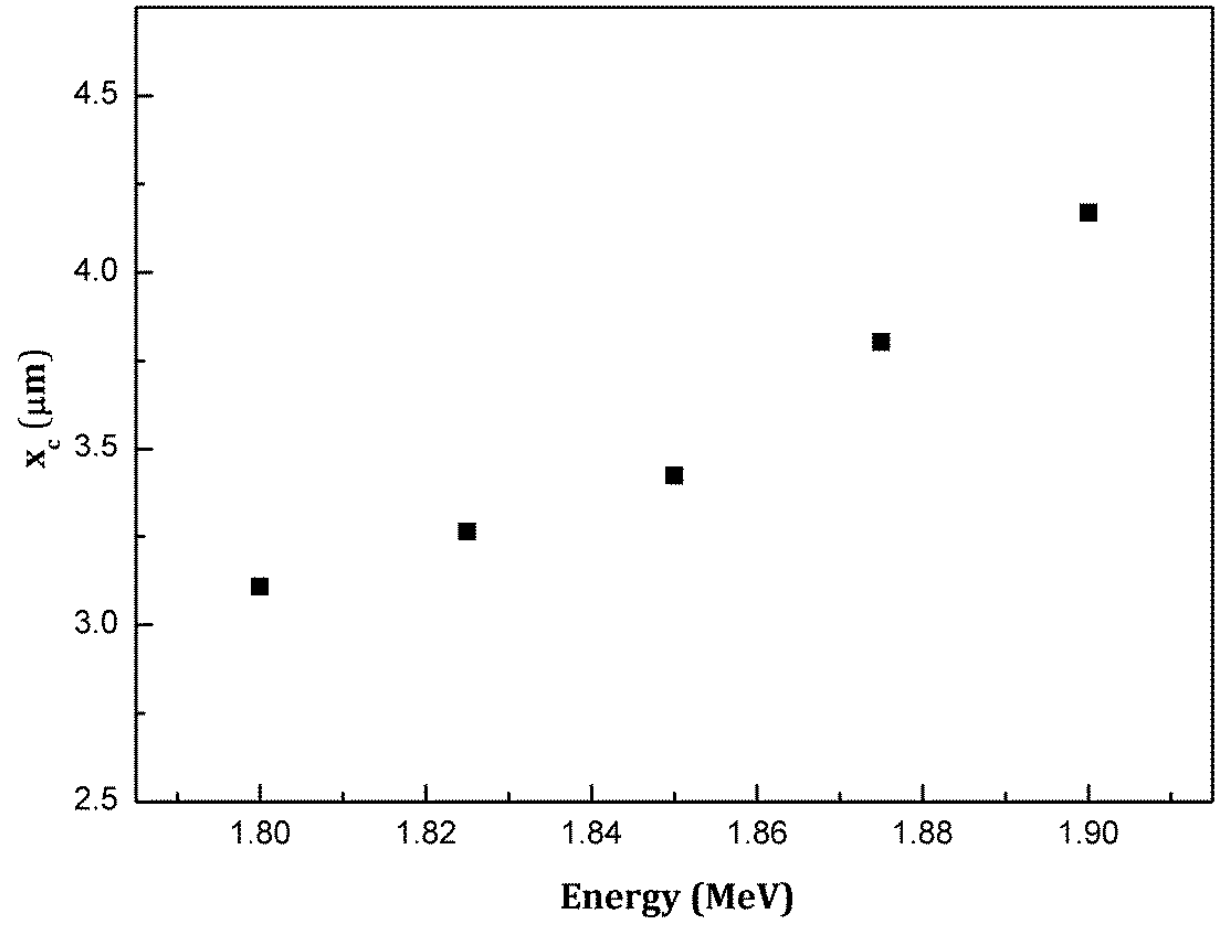

Fig. 7 